\title{
Longitudinal Magnetic Resonance Imaging Study of Cortical Development through Early Childhood in Autism
}

\author{
Cynthia M. Schumann, ${ }^{1,5}$ Cinnamon S. Bloss, ${ }^{6}$ Cynthia Carter Barnes, ${ }^{1}$ Graham M. Wideman, ${ }^{1}$ Ruth A. Carper, ${ }^{2}$ \\ Natacha Akshoomoff, ${ }^{2,3}$ Karen Pierce, ${ }^{1}$ Donald Hagler, ${ }^{4}$ Nicholas Schork, ${ }^{6,7}$ Catherine Lord, ${ }^{8}$ and Eric Courchesne ${ }^{1}$ \\ ${ }^{1}$ Autism Center of Excellence, Department of Neurosciences, ${ }^{2}$ Center for Human Development, and Departments of ${ }^{3}$ Psychiatry and ${ }^{4}$ Radiology, University \\ of California, San Diego, La Jolla, California 92093, ${ }^{5}$ M.I.N.D. Institute, Department of Psychiatry, University of California, Davis, Sacramento, California \\ 95817, ${ }^{6}$ The Scripps Translational Science Institute, Scripps Health, and 'Department of Molecular and Experimental Medicine, Scripps Research Institute, La Jolla, \\ California 92037, and ${ }^{8}$ Department of Psychology, Autism and Communication Disorders Center, University of Michigan, Ann Arbor, Michigan 48109
}

Cross-sectional magnetic resonance imaging (MRI) studies have long hypothesized that the brain in children with autism undergoes an abnormal growth trajectory that includes a period of early overgrowth; however, this has never been confirmed by a longitudinal study. We performed the first longitudinal study of brain growth in toddlers at the time symptoms of autism are becoming clinically apparent using structural MRI scans at multiple time points beginning at 1.5 years up to 5 years of age. We collected 193 scans on 41 toddlers who received a confirmed diagnosis of autistic disorder at $\sim 48$ months of age and 44 typically developing controls. By 2.5 years of age, both cerebral gray and white matter were significantly enlarged in toddlers with autistic disorder, with the most severe enlargement occurring in frontal, temporal, and cingulate cortices. In the longitudinal analyses, which we accounted for age and gender effect, we found that all regions (cerebral gray, cerebral white, frontal gray, temporal gray, cingulate gray, and parietal gray) except occipital gray developed at an abnormal growth rate in toddlers with autistic disorder that was mainly characterized by a quadratic age effect. Females with autistic disorder displayed a more pronounced abnormal growth profile in more brain regions than males with the disorder. Given that overgrowth clearly begins before 2 years of age, future longitudinal studies would benefit from inclusion of even younger populations as well as further characterization of genetic and other biomarkers to determine the underlying neuropathological processes causing the onset of autistic symptoms.

\section{Introduction}

Autism is a heterogeneous neurodevelopmental disorder of unknown etiology and poorly understood neuropathology. Children are given an autistic disorder diagnosis if they display three core features by three years of age: (1) impairments in reciprocal social interactions; (2) abnormal language development; and (3) repetitive and ritualized behaviors (American Psychiatric Association, 1994). Autism is diagnosed more often in boys than girls, at a ratio of 4:1 (Fombonne, 2003), and the cause(s) of the disorder, clinical characteristics, and neuroanatomical profiles may differ as well. A hypothesis has been proposed that the brain in toddlers with autism undergoes an

Received Nov. 17, 2009; revised Jan. 4, 2010; accepted Feb. 8, 2010.

This research program was primarily supported by funds from the National Institute of Neurological Disorders and Stroke (Grant 2-ROI-NS19855) and the Autism Center of Excellence (National Institutes of Health Grant 1-P50MH081755). We are grateful to the children and their families for years of dedication to this research program. In addition, we appreciate the assistance of Clare Roepke, Trudy Kao, and Ari Dubin in image collection and processing. We also thank Drs. Jody Buckwalter and John Morgan for helpful comments on the anatomical analyses and manuscript, respectively. We appreciate the contribution of Dr. Richard Haas in performing patient neurological assessment at Rady Children's Hospital, San Diego, California.

Correspondence should be addressed to either of the following: Dr. Cynthia Schumann, The M.I.N.D. Institute Department of Psychiatry and Behavioral Sciences, University of California Davis School of Medicine, 2805 50th Street, Sacramento, CA 95817, E-mail: cmschumann@ucdavis.edu; or Dr. Eric Courchesne, Autism Center of Excellence, Department of Neuroscience, University of California San Diego School of Medicine, La Jolla, CA 92093, E-mail: ecourchesne1949@gmail.com.

DOI:10.1523/JNEUROSCI.5714-09.2010

Copyright $\odot 2010$ the authors $\quad 0270-6474 / 10 / 304419-09 \$ 15.00 / 0$ abnormal growth trajectory that includes a period of early overgrowth (Courchesne et al., 2001). Autism is often diagnosed in the fourth year of life, yet cross-sectional magnetic resonance imaging (MRI) and head circumference studies suggest that early brain overgrowth occurs before the diagnosis is typically given.

At birth, head circumference in infants who later develop autism is typically near normal (Lainhart et al., 1997; Courchesne et al., 2007). However, by two years of age, head circumference becomes abnormally enlarged in children with autism (Courchesne et al., 2003; Dementieva et al., 2005; Hazlett et al., 2005; Dawson et al., 2007; Mraz et al., 2007; Webb et al., 2007), which corresponds to the age at which symptoms often become apparent. By the time children with autism reach 3 or 4 years of age, crosssectional MRI studies find that brain volume is abnormally enlarged by $\sim 5-10 \%$ (Courchesne et al., 2001; Carper et al., 2002; Sparks et al., 2002; Hazlett et al., 2005). A meta-analysis of all published head circumference and cross-sectional MRI brain size data through early 2005 showed that the period of greatest brain enlargement in autism is during the toddler years (Redcay and Courchesne, 2005), although it is unclear whether this is attributable to increases in cortical gray or white matter, or both, as reported in early cross-sectional MRI studies (Courchesne et al., 2001; Carper et al., 2002). Furthermore, there appears to be a regional gradient of abnormal enlargement by early childhood, whereby the frontal and temporal lobes are more affected, which parallels those regions mediating cognitive functions that tend to 
be most impaired (Carper et al., 2002; Hazlett et al., 2005; Bloss and Courchesne, 2007; Courchesne et al., 2007). There is, however, heterogeneity in both the severity of symptoms and regions of abnormal brain growth in children with an autism spectrum disorder (Amaral et al., 2008), particularly between males and females (Bloss and Courchesne, 2007).

Although the hypothesis of early brain overgrowth is currently one of the most prominent theories on the neuropathology of autism, the evidence comes from head circumference and crosssectional MRI studies and therefore awaits confirmation by a longitudinal MRI study of brain volume growth during the time when the symptoms of autism become clinically apparent. The goals of this research program were to (1) identify toddlers who displayed signs of developing autism and (2) use structural MRI at multiple time points through early development to identify which cortical regions show aberrant growth patterns.

\section{Materials and Methods}

Diagnostic assessment. One hundred eighteen toddlers ( 87 males, $31 \mathrm{fe}-$ males) began the study between the ages of 12 and 48 months (mean, $30 \pm 10$ months). Provisionally autistic children (e.g., children whose behavioral symptoms put them at risk for an autism spectrum disorder; see below) were recruited by clinician referral, through presentations at local autism support group meetings, and by a letter distributed to agencies (directed to parents of children with autism who may have younger siblings). Typically developing young children were recruited via notices given to local preschools, magazine advertisements, and referral from parents already participating in the program. A parent or guardian for each participant gave informed consent to participate in this research program as approved by a joint Institutional Review Board of Rady Children's Hospital San Diego and the University of California, San Diego. A requirement for participation in the study was that all children were full term at birth with no major complications during pregnancy or birth.

At entry into the study, children first received a clinical psychometric and diagnostic evaluation by a research psychologist (C.C.B. or N.A.). Children were reevaluated every $6-12$ months until they exited the study between 48 and 60 months of age, or voluntarily left the study before that age. At the first clinical visit and at each subsequent visit, either the Mullen Scales of Early Learning (Mullen, 1995) or the Wechsler Preschool and Primary Scale of Intelligence-III (Weschler, 2002) was administered to each participant as a standardized measure of early cognition. Toddlers (55 males, 19 females) who displayed symptoms of autism spectrum disorder, as determined by the research psychologist with extensive clinical diagnostic experience with autism, were assigned to a "provisional autism spectrum disorder" (p-ASD) group. These children were also given the Autism Diagnostic Observation Schedule-G (ADOS-G) (Lord et al., 2000) at entry and at subsequent visits, and their parents completed the Autism Diagnostic Interview (ADI-R) (Lord et al., 1994) at entry and exit clinical visits. The ADOS-G is a semistructured standardized assessment of communication, social interaction, and play for young children to evaluate presence of an autism spectrum disorder. It provides a diagnostic classification of autistic disorder, autism spectrum, or nonspectrum based on the child's presentation during the assessment. The ADI-R is a standardized comprehensive parent interview administered by a trained clinician using a semistructured interview format. Questions on this measure are designed to elicit relevant information to assess, confirm, or rule out an autism spectrum disorder through queries closely associated with the diagnostic criteria set forth in the DSM-IV (Diagnostic and Statistical Manual of Mental Disorders-IV) (American Psychiatric Association, 1994). Typically developing toddlers, who showed no history of any developmental delay and were judged as typically developing after behavioral and psychometric testing by the clinician, were included in the "control" group ( 32 males, 12 females). The Vineland Adaptive Behavioral Scales Survey Interview (Sparrow et al., 1984), a standardized parent or caregiver interview designed to asses a child's functional skills in four different
Table 1. Subject and diagnostic data at final clinical visit

\begin{tabular}{|c|c|c|c|c|}
\hline & \multicolumn{2}{|c|}{$\begin{array}{l}\text { Autistic } \\
\text { disorder }(n=41)\end{array}$} & \multicolumn{2}{|c|}{ Typical $(n=44)$} \\
\hline & Male & Female & Male & Female \\
\hline Number of subjects & 32 & 9 & 32 & 12 \\
\hline Age range at MRI (months) & $22-67$ & $26-58$ & $12-63$ & $12-61$ \\
\hline Mean age at final clinical visit (months) & $49 \pm 1$ & $49 \pm 10$ & $44 \pm 3$ & $46 \pm 1$ \\
\hline Full-scale IQ & $57 \pm 18$ & $57 \pm 23$ & $111 \pm 16$ & $116 \pm 15$ \\
\hline Verbal IQ & $50 \pm 19$ & $50 \pm 29$ & $112 \pm 17$ & $116 \pm 15$ \\
\hline Performance IQ & $63 \pm 19$ & $62 \pm 21$ & $107 \pm 20$ & $115 \pm 16$ \\
\hline ADI-R Social & $18 \pm 5$ & $18 \pm 6$ & $\mathrm{n} / \mathrm{a}$ & $\mathrm{n} / \mathrm{a}$ \\
\hline ADI-R NV Comm. & $9 \pm 3$ & $9 \pm 3$ & $\mathrm{n} / \mathrm{a}$ & $\mathrm{n} / \mathrm{a}$ \\
\hline ADI-R RnR & $6 \pm 2$ & $5 \pm 2$ & $\mathrm{n} / \mathrm{a}$ & $\mathrm{n} / \mathrm{a}$ \\
\hline Vineland Social & $66 \pm 9$ & $64 \pm 7$ & $97 \pm 8$ & $102 \pm 15$ \\
\hline Vineland Comm. & $66 \pm 11$ & $62 \pm 16$ & $108 \pm 11$ & $105 \pm 9$ \\
\hline Vineland ABC & $63 \pm 8$ & $64 \pm 11$ & $100 \pm 10$ & $106 \pm 12$ \\
\hline
\end{tabular}

n/a, Not applicable; NV Comm., nonverbal communication; RnR, restricted and repetitive.

Table 2. Number of successful scans per subject by diagnostic group and the average number and range of months between scans

\begin{tabular}{lcccc}
\hline $\begin{array}{l}\text { Number } \\
\text { of scans }\end{array}$ & $\begin{array}{l}\text { Autistic } \\
\text { disorder }\end{array}$ & Typical & $\begin{array}{l}\text { Mean number and } \\
\text { range of months } \\
\text { between scans }\end{array}$ & $\begin{array}{l}\text { Total number } \\
\text { of scans }\end{array}$ \\
\hline 1 & 15 & 12 & & 27 \\
2 & 16 & 14 & $17(7-25)$ & 60 \\
3 & 6 & 8 & $10(5-19)$ & 42 \\
4 & 3 & 5 & $8(3-19)$ & 32 \\
5 & 0 & 4 & $9(5-20)$ & 20 \\
6 & 1 & 1 & $5(0-8)$ & 12 \\
Total number of subjects & 41 & 44 & & 193 \\
\hline
\end{tabular}

developmental domains, was also administered at intake and at subsequent visits for control subjects and for most children in the p-ASD group.

At $\geq 48$ months of age, children in the $\mathrm{p}$-ASD group were given a final diagnosis of autistic disorder ( 32 males, 9 females), pervasive developmental disorder-not otherwise specified (PDD-NOS; 8 males, 3 females), or developmental delay/nonspectrum (10 males, 5 female) based on the exit ADI-R administered by C.C.B. or N.A. and the exit ADOS-G administered by C.L. and either C.C.B. or N.A. Seven toddlers ( 5 males, 2 females) dropped from the p-ASD group before final assessment could be given. Only MRI data from typically developing controls and from children who received a final diagnosis of autistic disorder via this procedure were analyzed for this report (see Table 1 for diagnostic data from final clinical visit)

Neuroimaging. After the first clinical visit and after each subsequent visit, an MRI scan was collected at the UCSD Medical Center, Hillcrest on a 1.5 tesla Siemens Symphony system. A parent or guardian for each participant signed consent before the child entered the MRI scanner and was present throughout the duration of the scan in an adjacent waiting room. Forty-six p-ASD participants required anesthesia to undergo MRI. All other children were scanned at night during natural sleep. The protocols for scanning each participant included a three-dimensional T1weighted magnetization-prepared rapid gradient echo [MPRAGE; 128 coronal slices; voxel size, $0.859375 \times 0.859375 \times 1.5 \mathrm{~mm}$; echo time (TE), $3.67 \mathrm{~ms}$; repetition time (TR), $2730 \mathrm{~ms}$ ] and a constructive interference steady state (CISS; 128 coronal slices; voxel size, $0.859375 \times$ $0.859375 \times 1.5 \mathrm{~mm}$; TE, $3.08 \mathrm{~ms}$; TR, $6.15 \mathrm{~ms}$ ). A total of 193 scans was collected and analyzed for this study; the number of scans subjects received is listed in Table 2 .

Scans were transferred to the laboratory where processing consisted of a combination of semimanual anatomist-guided steps using in-house software plus the program Freesurfer. First, laboratory-developed visual registration software was used to align the MPRAGE and CISS image sets. Then, semiautomated laboratory-developed software using the CISS image (which emphasizes tissue-fluid contrast) was applied to remove nonbrain structures (e.g., skull) from MPRAGE and CISS image sets. The 


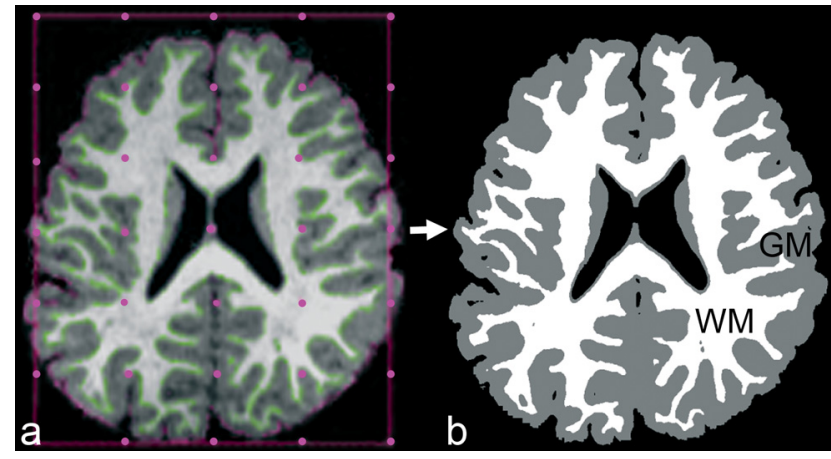

Figure 1. Axial section as shown in GWseg, displaying semimanual local threshold segmentation of cerebrum into cerebral gray matter (GM) and cerebral white matter (WM) (a) and resulting tissue classification used by Freesurfer to determine gray/white surface $(\boldsymbol{b})$.

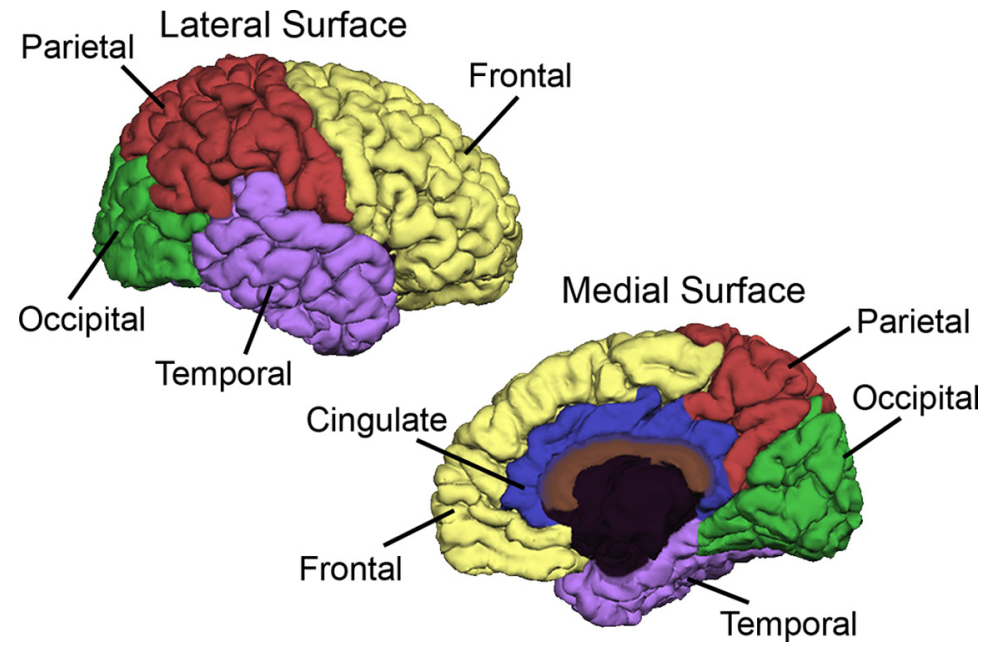

Figure 2. Surface parcellation of cerebral gray (adapted from Desikan et al., 2006).

program AFNI (Cox, 1996) was then used to align both image sets along the anterior commissure to posterior commissure axis. Both image sets were then imported into a laboratory-developed (by G.M.W.) semimanual segmentation program, GWseg, which permitted anatomists to guide voxel classification into gray matter, white matter, and nonbrain/CSF using a combination of global and local contrast thresholds. Specifically, for a subset of slices, the software displays each slice with a grid of threshold adjustment "knobs" (e.g., $7 \times 5$ ) (Fig. 1) overlaid onto the slice; the anatomist adjusts the threshold knobs to obtain the optimal tissue boundary for each slice. Thresholds for intervening slices are interpolated. Intrarater and interrater reliability (ICC) of at least $95 \%$ on 30 cases was established by each anatomist who used the segmentation program to classify tissue types for this study.

The boundaries of the cerebrum were manually defined as previously described in detail (Courchesne et al., 2001; Carper et al., 2002) (in which subcortical brain structures were excluded) using the laboratorydeveloped software program AREA (Egaas et al., 1995) (AREA-MRI measurement tool, (C1996 by M. Belmonte; based on a problem definition by S. Hinds and B. Egaas, University of California San Diego, La Jolla, CA). These boundaries were then overlaid onto the tissue-segmented/ classified image, and total cerebral, cerebral gray, and cerebral white matter volumes were measured. An ICC of at least $95 \%$ was established by each anatomist on all manual tracings.

To obtain lobe volume measures from the total cerebral gray volume, the MPRAGE image set for each subject was imported into the automated program Freesurfer 4.0 (Dale et al., 1999; Fischl et al., 1999, 2001) with some modifications as described below. The program normally works by first defining a white matter surface from the MPRAGE images. However, because of the low contrast of gray and white matter in infant and toddler brains, which are not as well myelinated as adult brains, this initial Freesurfer step often failed to produce an adequate white matter surface. We addressed this problem by substituting our reliable anatomist-guided GWseg-based segmentation (described above) for the Freesurfer white surface (wm.mgz), which allowed Freesurfer to proceed with the remaining automated steps of finding the outer gray/pial surface, as described in detail previously (Dale et al., 1999; Fischl et al., 1999, 2001 ). Once the gray and white matter surface was determined, cortical surface parcellations were derived from an automated labeling system in Freesurfer that subdivides the cerebral cortex into 34 neuroanatomical subregions per hemisphere. A detailed description of the development and validation of this labeling system was provided previously (Desikan et al., 2006). The goal of this study was to report cerebral lobe volumes; therefore, we assigned each of the 34 subregions to the appropriate cerebral lobe category so that each gray matter voxel had an associated lobe label. To insure that all gray matter voxels were completely and accurately labeled, the lobe labels from Freesurfer were then overlaid back onto the original anatomist-guided segmentation images to produce volume measures for frontal, temporal, parietal, occipital, and cingulate cortices (Figs. 2, 3).

Statistical analyses. All statistical analyses were performed using the SPSS 14.0 and 16.0 (SPSS). We first performed cross-sectional analyses of volumetric data for each region of interest (ROI) measured from a single timepoint scan collected from each subject at $\sim 2.5$ years of age. This age was chosen by selecting the earliest scan collected for each autistic disorder group subject; computing the average age, which resulted in 32 months; and selecting the scan collected from each control subject closest to 32 months of age. We then performed a general linear model analysis of covariance (ANCOVA) for each ROI to compare volumes between autistic disorder and control subjects at 2.5 years of age covarying for age at scan and gender.

Then, because of the longitudinal nature of the study and the fact that the data consist of nonuniform numbers of repeated measurements at nonuniform ages and intervals, we used linear mixed-effects model analysis. In the linear mixed-effects model used, the intercept term is treated as a random effect that varies by individual such that intraindividual correlation among the measures collected on a particular individual, $\varepsilon$, is taken into account. We then proceeded with longitudinal analyses using linear mixed-effects models to account for the presence of repeated measurements on individual subjects. It is important to keep in mind that the central aim of this study was to determine whether longitudinal patterns of cerebral gray and white matter growth in autistic and typical toddlers are consistent with age-related changes that have been reported previously in cross-sectional studies of MRI neuroanatomy in early life in autistic disorder. Therefore, although we used linear mixedeffects models to account for the presence of repeated measurements on individual subjects, we based our choice of independent variables on the models used in these previous reports (Courchesne et al., 2001; Carper et al., 2002). Specifically, we used the following six variables as independent variables or predictors of volume for different ROIs: gender, diagnosis (dummy coded), linear age, quadratic age, interaction between diagnosis and linear age, and interaction between diagnosis and quadratic age. All age terms were centered on the grand mean to allow ease of interpretation of the regression coefficients and to stabilize the analysis by decreasing multicollinearity among otherwise highly correlated main-effect predictor variables (e.g., linear age and quadratic age), as well as between main effects and interaction terms (e.g., linear age and linear age by diagnosis) (Cohen et al., 2003). Particular emphasis was placed on the linear and quadratic effects of age on brain size, since they are likely to reflect growth trajectory characteristics with respect to which children with autistic disorder may differ from typically developing children. A 


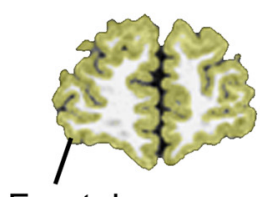

Frontal
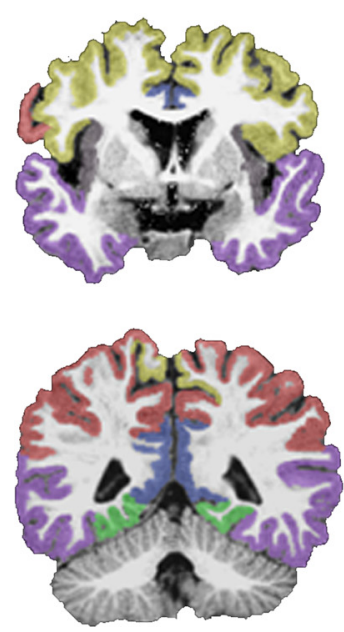

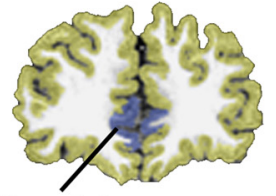

Cingulate
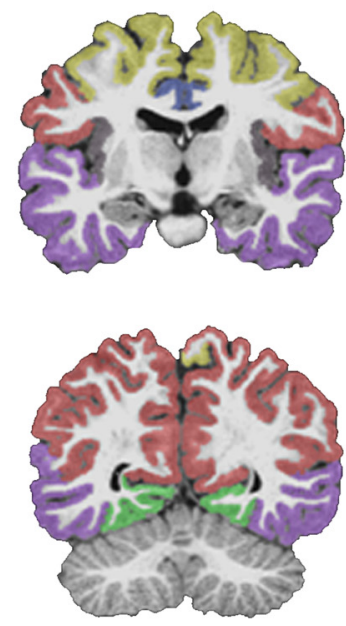
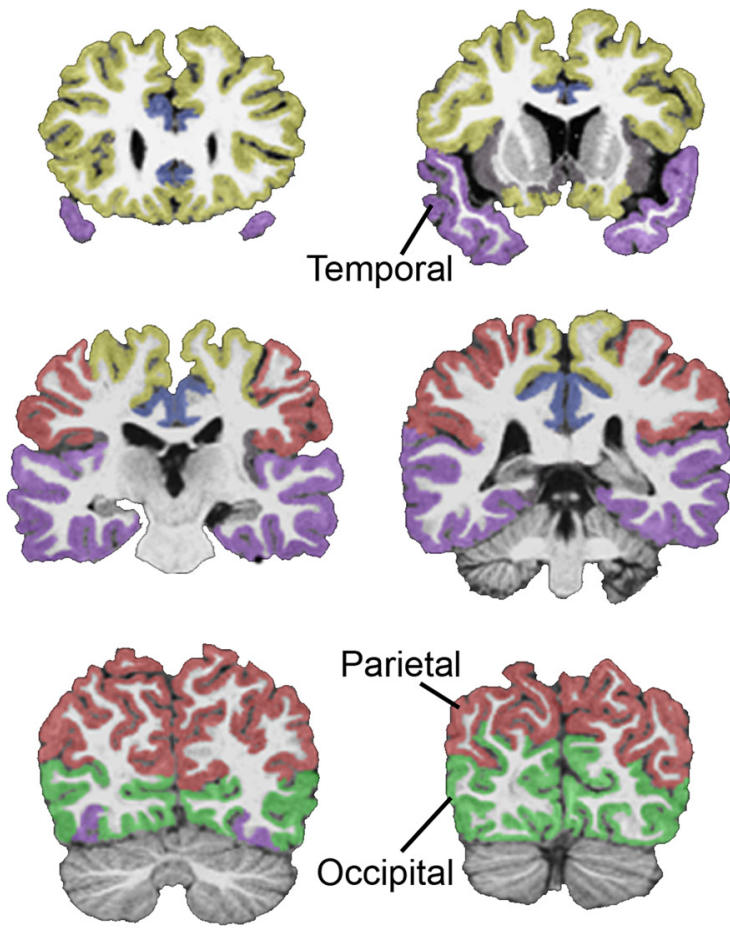

Figure 3. Coronal images indicating parcellation boundaries for lobe regions of cerebral gray.

total of eight ROIs, including frontal gray, temporal gray, parietal gray, occipital gray, cingulate gray, total gray, total white, and total cerebral volume were analyzed in this manner. Furthermore, three different contrasts were tested for statistical significance: autistic disorder versus typical subjects, autistic disorder males versus typical males, and autistic disorder females versus typical females. Gender, as indicated above, was included as a covariate in models that included both male and female subjects.

The current study and previous cross-sectional MRI study reports on autistic toddlers indicate a unidirectional a priori hypothesis of abnormal enlargement, and not reduction, in cerebral gray and white matter volumes. Therefore, in determining statistical significance, we used an $\alpha$ level of 0.1 in the interpretation of regression coefficients reflecting the effect of diagnosis for those ROIs found to be associated with diagnosis in the previous studies. An $\alpha$ level of 0.05 was used in the interpretation of regression coefficients that represent the effect of diagnosis in tests of ROIs that have not previously been examined (e.g., cingulate gray matter volume).

\section{Results}

\section{Diagnostic measures}

Clinical diagnostic and behavioral measures collected at the final clinical visit ( $>48$ months of age for autistic disorder subjects) are listed in Table 1. There was, as expected, a significant group effect for all intelligence quotient and Vineland measures $(p<0.001)$. Table 2 lists the number of MRI scans collected per subject by diagnostic group.

\section{Cross-sectional volumetric analyses of males and females at} 2.5 years of age

ANCOVA (Table 3) for all subjects, covarying for gender and age at scan, revealed significantly increased total cerebral volume $\left(F_{(1,82)}=5.79 ; p=0.018\right)$ in the autistic disorder group relative to controls by $7 \%$. Total cerebral white matter volume $\left(F_{(1,82)}=3.95 ; p=0.050\right)$ and gray matter volume $\left(F_{(1,82)}=\right.$ $4.09 ; p=0.046)$ were significantly larger in the autistic disorder group relative to controls by 10 and 5\%, respectively.
Table 3. Cross-sectional volumetric analyses (ANCOVA with gender and age at MRI scan as covariates) at $\sim 2.5$ years of age for combined sample of males and females with autistic disorder compared with typically developing controls

\begin{tabular}{lcclc}
\hline & $\begin{array}{l}\text { Autistic } \\
\text { disorder }(n=41)\end{array}$ & $\begin{array}{l}\text { Control } \\
(n=44)\end{array}$ & $\begin{array}{l}\text { Significance } \\
(p \text { value })\end{array}$ & $\begin{array}{l}\text { Percentage } \\
\text { difference }\end{array}$ \\
\hline Mean age at MRI (months) & $32 \pm 9$ & $30 \pm 11$ & 0.182 & \\
Total cerebrum $\left(\mathrm{cm}^{3}\right)$ & $984 \pm 76$ & $920 \pm 85$ & $0.018^{*}$ & $7 \%$ \\
Total white $\left(\mathrm{cm}^{3}\right)$ & $307 \pm 32$ & $277 \pm 47$ & $0.050^{*}$ & $10 \%$ \\
Total gray $\left(\mathrm{cm}^{3}\right)$ & $676 \pm 59$ & $643 \pm 54$ & $0.046^{*}$ & $5 \%$ \\
Frontal gray $\left(\mathrm{cm}^{3}\right)$ & $232 \pm 24$ & $218 \pm 19$ & $0.038^{*}$ & $6 \%$ \\
Temporal gray $\left(\mathrm{cm}^{3}\right)$ & $142 \pm 14$ & $131 \pm 13$ & $0.008^{* *}$ & $9 \%$ \\
Cingulate gray $\left(\mathrm{cm}^{3}\right)$ & $26 \pm 3$ & $24 \pm 3$ & 0.097 & $8 \%$ \\
Parietal gray $\left(\mathrm{cm}^{3}\right)$ & $165 \pm 15$ & $160 \pm 15$ & 0.289 & $4 \%$ \\
Occipital gray $\left(\mathrm{cm}^{3}\right)$ & $80 \pm 8$ & $78 \pm 9$ & 0.225 & $3 \%$ \\
\hline
\end{tabular}

${ }^{*} p \leq 0.05 ;{ }^{*} p \leq 0.01$.

Frontal gray $\left(F_{(1,82)}=4.43 ; p=0.038\right)$ and temporal gray $\left(F_{(1,82)}=7.37 ; p=0.008\right)$ matter volumes were also significantly larger in the autistic disorder group relative to controls by 6 and $9 \%$, respectively. Cingulate volume was $8 \%$ larger in children with autistic disorder relative to controls, but this did not reach statistical significance in our sample. There was no difference in the volumes of parietal or occipital gray at $\sim 2.5$ years of age.

\section{Longitudinal analyses of males and females}

Table 4 depicts the results of our best-fit linear mixed-effects model analyses for the combined sample of males and females for each ROI volume measured. We started from a complete general linear mixed-effects model with all available terms, and then higher-order terms that were nonstatistically significant were removed in a stepwise manner until the best-fit model was reached. For all regions except frontal and occipital gray matter volumes, the general linear mixed-effects model was the best-fit model. For total cerebral volume, there were main effects of 
Table 4. Results of best-fit mixed-effects model analyses in a combined sample of males and females with autistic disorder compared with typically developing controls

\begin{tabular}{|c|c|c|c|c|c|c|}
\hline Region & Gender & Linear age & Diagnosis & Diagnosis $\times$ linear age & Quadratic age & Diagnosis $\times$ quadratic age \\
\hline Total cerebrum & $0.003^{a}$ & $<0.0001^{a}$ & 0.222 & 0.546 & $<0.0001^{a}$ & $<0.001^{a}$ \\
\hline Total white & 0.111 & $<0.0001^{a}$ & 0.713 & 0.264 & $<0.0001^{a}$ & $0.051^{a}$ \\
\hline Total gray & $0.002^{a}$ & $<0.0001^{a}$ & 0.133 & 0.839 & $0.002^{a}$ & $0.073^{a}$ \\
\hline Frontal gray & $0.002^{a}$ & $<0.0001^{a}$ & $0.027^{a}$ & & & \\
\hline Temporal gray & $0.007^{a}$ & $<0.0001^{a}$ & $0.008^{a}$ & 0.972 & 0.062 & $0.058^{a}$ \\
\hline Cingulate gray & 0.120 & $<0.0001^{a}$ & 0.464 & 0.959 & $<0.0001^{a}$ & $0.010^{a}$ \\
\hline Parietal gray & 0.053 & $<0.0001^{a}$ & 0.647 & 0.352 & $0.008^{a}$ & $0.027^{a}$ \\
\hline Occipital gray & $0.001^{a}$ & $<0.0001^{a}$ & 0.620 & & $0.009^{a}$ & \\
\hline
\end{tabular}

Bold indicates a significant difference between autistic disorder and control.

asignificant effect.

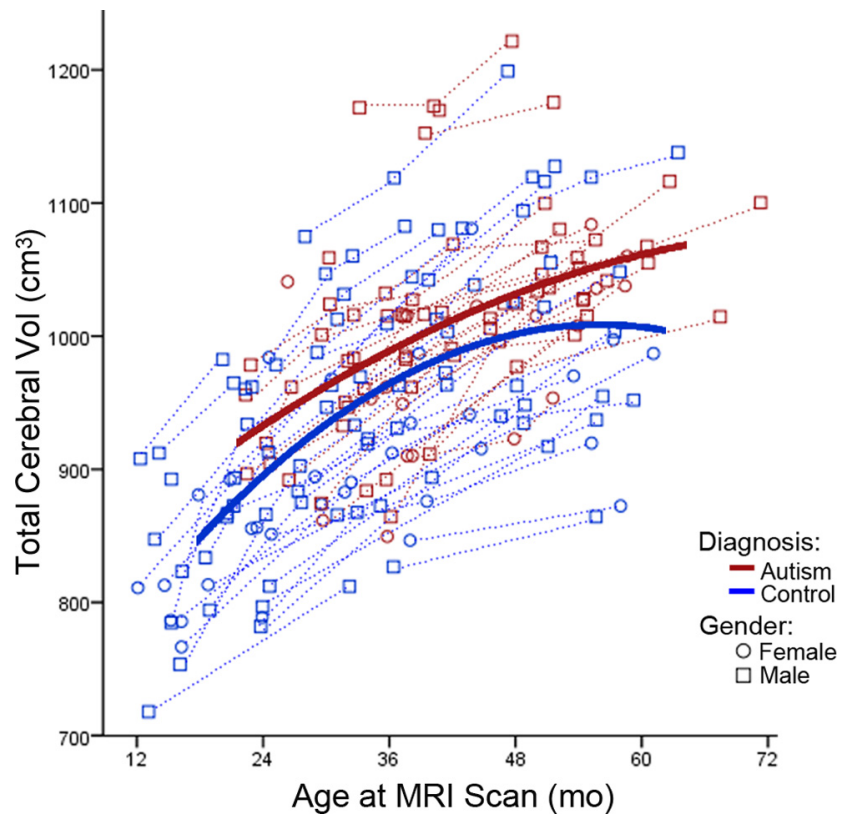

Figure 4. Total cerebral volume (Vol) growth trajectory for males $(\bigcirc)$ and females ( $\square$ ). Dotted lines represent each subject's volume measurements; solid lines are autistic disorder (red) and typical control (blue) group quadratic growth regression curves. mo, Months.

gender $(p=0.003)$, linear age $(p<0.0001)$, and quadratic age $(p<0.0001)$, and the interaction between quadratic age and diagnosis $(p<0.0001)$ indicated a significant difference in growth trajectory between autistic disorder and control (Fig. 4). For total white matter volume, there were main effects of linear age $(p<0.0001)$ and quadratic age $(p<0.0001)$, as well as an interaction between diagnosis and quadratic age $(p=0.051)$, indicating the effect of quadratic age significantly varied between autistic disorder and control children (Fig. 5). For total gray matter volume, there were main effects of gender $(p=0.002)$, linear age $(p<0.0001)$, and quadratic age $(p<0.002)$, and the interaction between quadratic age and diagnosis $(p=0.073)$, which indicates a significant difference in growth trajectory between autistic disorder and control (Fig. 5). Temporal gray matter showed main effects of gender $(p=0.007)$, linear age $(p<$ $0.0001)$, and diagnosis $(p=0.008)$, and the effect of quadratic age as an interaction with diagnosis $(p=0.058)$ significantly varied between autistic disorder and control (Fig. 6). Parietal and cingulate gray matter volumes both showed main effects of linear age $(p<0.0001)$ and quadratic age $(p=0.008$ and $p<0.0001$, respectively) and an interaction between quadratic age by diagnosis $(p=0.008$ and $p<0.0001$, respectively), which indicated a significant difference in the growth trajectories of both regions between autistic disorder and control.

With respect to frontal gray matter, the best-fitting model was one that included the main effects of gender $(p=0.002)$, linear age $(p<0.0001)$, and diagnosis $(p=0.027)$, indicating that frontal gray matter volumes significantly differed between autistic disorder and control (Fig. 6). For occipital gray matter, the best-fitting model was one that included the main effects of gender $(p=0.0008)$, linear age $(p=0.038)$, diagnosis $(p=0.62)$, and quadratic age $(p=0.009)$, indicating no significant differences between autistic disorder and control in either volume or growth pattern in occipital cortex.

\section{Longitudinal analyses of males}

Table 5 depicts the results of our best-fit linear mixed-effects model analyses for males only for each ROI volume measured. For total cerebral volume, the best-fitting model included main effects of linear age $(p<0.0001)$, diagnosis $(p=0.43)$, quadratic age $(p<0.0001)$, and an interaction between quadratic age and diagnosis $(p<0.009)$, which indicated a significant difference in growth trajectory between autistic disorder and control males. For total white matter volume, the best-fitting model included main effects of linear age $(p<0.0001)$, diagnosis $(p=0.71)$, and quadratic age $(p<0.0001)$. For total gray matter volume, the best-fitting model included only main effects of linear age $(p<$ $0.0001)$ and diagnosis $(p=0.11)$. Thus, only total cerebral volume, but not gray and white matter volumes, showed differences between male autistic disorder and control children in growth trajectory.

With respect to both frontal and temporal gray matter volumes, the best-fitting models included main effects of linear age (frontal, $p<0.0001$; temporal, $p<0.0001$ ) and diagnosis (frontal, $p=0.081$; temporal, $p=0.018$ ), indicating significant differences in volumes between male autistic disorder and control subjects in both of these regions. For parietal and cingulate gray matter volumes, the best-fit models included main effects of linear age (parietal, $p=0.001$; cingulated, $p<0.0001$ ), diagnosis (parietal, $p=0.91$; cingulated, $p=0.97$ ), and quadratic age (parietal, $p=0.14$; cingulated, $p<0.0001$ ), as well as the interactions between diagnosis and linear age (parietal, $p=0.17$; cingulated, $p=0.76$ ) and diagnosis and quadratic age (parietal, $p=0.084$ cingulated, $p=0.030$ ), indicating the effect of quadratic age on the growth trajectory of each region significantly varied between autistic disorder and control males. For occipital gray matter volume, the best-fitting model included main effects of both linear age $(p=0.21)$ and diagnosis $(p=0.41)$, revealing no difference between autistic disorder and control males in either volume or growth trajectory in occipital cortex. 
Longitudinal analyses of females

Table 6 depicts the results of our best-fit linear mixed-effects model analyses for females only for each ROI volume measured. For total cerebral volume, the best-fitting model was one that included main effects of linear age $(p<0.0001)$, diagnosis $(p=0.27)$, quadratic age $(p<$ $0.0001)$, as well as the interaction between diagnosis and linear age $(p=0.029)$ and an interaction between quadratic age and diagnosis $(p<0.019)$, which indicated a significant difference in growth trajectory between autistic disorder and control females. With respect to total white matter volume, the best-fitting model included main effects of linear age $(p<0.0001)$, diagnosis $(p=0.48)$, and quadratic age $(p<0.0001)$, as well as interactions between diagnosis and linear age $(p=$ $0.001)$ and diagnosis and quadratic age $(p=0.002)$, indicating the effects of both linear and quadratic age on white matter growth significantly varied between female autistic disorder and control children. For total gray matter volume, the best-fitting model included main effects of linear age $(p=0.0001)$, diagnosis $(p=$ $0.23)$, quadratic age $(p=0.002)$, and an interaction between diagnosis and linear age $(p=0.021)$, which indicated a significant difference in the growth trajectory between female autistic disorder and control children.

With respect to both frontal and parietal gray matter volumes, the best-fitting models included main effects of linear age (frontal, $p<0.0001$; parietal, $p=0.013$ ), diagnosis (frontal, $p=0.40$; parietal, $p=$ 0.32 ), and quadratic age (frontal, $p=$ 0.007; parietal, $p=0.033$ ), as well as the interaction between diagnosis and linear age (frontal, $p=0.010$; parietal, $p=0.096$ ), suggesting that the effect of linear age on the growth trajectories of both regions significantly varied between autistic disorder and control females. With respect to total temporal volume, the bestfitting model included main effects of linear age $(p<0.0001)$, diagnosis $(p=0.069)$, and quadratic age $(p=0.008)$, as well as interactions between diagnosis and linear age $(p=0.66)$ and diagnosis and quadratic age ( $p=0.078)$, indicating that temporal volumes, as well as the effects of both linear and quadratic age on the growth trajectory of temporal cortex, significantly varied between autistic disorder and control. For occipital and cingulate volumes, the selected models included main effects of linear age (occipital, $p=0.088$; cingulated, $p<0.0001$ ), diagnosis (occipital, $p=0.62$; cingulated, $p=0.052$ ), and quadratic age (occipital, $p=0.013$; cingulated, $p=0.001$ ), revealing a strong trend toward a significant difference in cingulate gray volume between autistic disorder and control, and no difference in either volume or growth pattern in occipital gray.

\section{Discussion}

In this first longitudinal study of cerebral cortical development in young children with autism, we provide evidence that the cerebrum and several of its subdivisions undergo an abnormal growth trajectory that includes overgrowth by 2.5 years of age at the time symptoms are becoming clinically evident. This study replicates and extends the findings of our own laboratory (Courchesne et al., 2001; Carper et al., 2002) as well as those of others (Sparks et al., 2002; Hazlett et al., 2005) that children with autism already have enlarged brains by this very young age. Both cerebral gray and white matter demonstrated significant growth abnormalities but also differed by region and gender. Within cortex, the most drastic differences in volume and age-related change occurred in the anterior regions of the brain, including frontal, temporal, and cingulate cortices; posterior cerebral regions were less affected in both volume and growth trajectory. In both the longitudinal and cross-sectional analyses, the most significant aberrant growth was in temporal gray, which also replicates previous findings (Sparks et al., 2002; Hazlett et al., 2005) that temporal gray is enlarged at $\sim 2.5$ years of age.

Courchesne et al. (2001) first reported an enlargement in cerebral white and gray matter in children with autism at 2 and 3 years of age, a phenomenon not observed in the older children and adolescents. Hazlett et al. (2005) and Sparks et al. (2002) replicated these findings, both reporting a significant increase in 
Table 5. Significant differences from best-fit model analyses of males with autistic disorder compared with typically developing controls

\begin{tabular}{|c|c|c|c|c|c|}
\hline Region & Linear age & Diagnosis & Diagnosis $\times$ linear age & Quadratic age & Diagnosis $\times$ quadratic age \\
\hline Total cerebrum & $<0.0001^{a}$ & 0.430 & 0.559 & $<0.0001^{a}$ & $0.009^{a}$ \\
\hline Frontal gray & $<0.0001^{a}$ & $0.081^{a}$ & & & \\
\hline Temporal gray & $<0.0001^{a}$ & $0.018^{a}$ & & & \\
\hline Cingulate gray & $<0.0001^{a}$ & 0.970 & 0.760 & $<0.0001^{a}$ & $0.030^{a}$ \\
\hline
\end{tabular}

Bold indicates a significant difference between autistic disorder and control.

asignificant effect.

Table 6. Significant differences from best-fit model analyses of females with autistic disorder compared with typically developing controls

\begin{tabular}{|c|c|c|c|c|c|}
\hline Region & Linear age & Diagnosis & Diagnosis $\times$ linear age & Quadratic age & Diagnosis $\times$ quadratic age \\
\hline Total cerebrum & $<0.0001^{a}$ & 0.273 & $0.029^{a}$ & $<0.0001^{a}$ & $0.019^{a}$ \\
\hline Total white & $<0.0001^{a}$ & 0.480 & $0.001^{a}$ & $<0.0001^{a}$ & $0.002^{a}$ \\
\hline Total gray & $<0.0001^{a}$ & 0.230 & $0.021^{a}$ & $0.002^{a}$ & \\
\hline Frontal gray & $<0.0001^{a}$ & 0.400 & $0.010^{a}$ & $0.007^{a}$ & \\
\hline Temporal gray & $<0.0001^{a}$ & $0.069^{a}$ & 0.660 & $0.008^{a}$ & $0.078^{a}$ \\
\hline Cingulate gray & $<0.0001^{a}$ & $0.052^{a}$ & & $0.001^{a}$ & \\
\hline
\end{tabular}

Bold indicates a significant difference between autistic disorder and control.

${ }^{a}$ Significant effect.

the size of the cerebrum relative to controls at $\sim 2-4$ years of age. Hazlett et al. (2005) subdivided the cerebrum into gray and white matter volumes and further replicated the report by Courchesne et al. (2001) by finding increases in both regions relative to controls at $\sim 2.5$ years of age. Carper et al. (2002) examined the same cohort of children as reported by Courchesne et al. (2001) and found that increases in cerebral gray matter were primarily attributed to increases in frontal and temporal cortices. That cross-sectional study predicted that the mean frontal and temporal lobe volumes in typically developing children were increasing by $\sim 20 \%$ from 2 to 8 years of age, whereas these regions only were increasing by about $1 \%$ in children with autism over the same age range. Our current within-subject longitudinal study not only confirmed that early brain overgrowth occurs but also confirmed the hypothesis that the autistic brain is continuing to undergo abnormal development through early childhood.

The anatomical delineations used in the current study differed from previous cross-sectional reports in that the anterior cingulate cortex was included as part of a cingulate gray measure, rather than as part of the frontal lobe as reported previously (Carper et al., 2002; Hazlett et al., 2005). This difference may have impacted the findings of the current study for frontal gray matter and likely accounts for differences in the level of significance between autism and control in this and previous reports (Carper et al., 2002). We also found a trend toward enlargement of cingulate gray in children with autism at 2.5 years of age in our present cross-sectional analyses and a significant enlargement in cingulate gray volume in females with autism in our longitudinal analyses, which likely accounts for the apparent discrepancy in frontal gray effect sizes between studies.

We observed significant gender differences in the longitudinal growth trajectories of several brain regions. In males with autism, frontal and temporal lobe gray matter volumes were significantly enlarged, and cingulate gray matter grew at a nonlinear rate that differed relative to controls. The abnormal brain growth in autistic females was more widespread and severe, with total cerebrum, cerebral white, cerebral gray, frontal, and temporal demonstrating abnormal growth trajectories relative to controls; in addition, cingulate gray was enlarged in females but not males with autism. Females with autism also continued to undergo an abnormal growth trajec- tory in cerebral white and gray matter compared with typically developing females, whereas males with the disorder did not. These results provide additional evidence for our previous hypothesis that males and females with autism may exhibit different neuroanatomical profiles, with females presenting a more pronounced pathology (Bloss and Courchesne, 2007; Schumann et al., 2009). The results are also analogous to our recently reported cross-sectional findings in the amygdala, in which differences between autistic and typically developing toddlers were more pronounced in females than in males (Schumann et al., 2009). It is possible that overgrowth may begin earlier in males than females with autism; thus, the age that we are observing in the current study is at the peak of abnormal development for females specifically. Alternatively, it is possible that females with autism display more severely impacted brain overgrowth. This prolonged growth trajectory may result in more severe cognitive and behavioral impairments (Bloss and Courchesne, 2007).

Our finding of cerebral enlargement by 2.5 years of age, a time when symptoms of autism are becoming clinically evident, begs the question of when overgrowth actually begins and what underlying neurobiological aberrancies contribute to continued abnormal growth. Head circumference studies imply that brain size is near normal at birth but becomes enlarged by 2 years of age (Courchesne et al., 2003; Dementieva et al., 2005; Hazlett et al., 2005; Dawson et al., 2007; Webb et al., 2007). During this time in development, the typically developing brain undergoes extensive dendritic arborization and active pruning of neuronal processes (Conel, 1939-1967; Huttenlocher, 1979). Genetic factors and/or overproduction of neurotrophins may lead to excessive cell proliferation and survival and/or exuberant dendritic branching producing cortical overgrowth. Insufficient synaptic pruning of connections between neurons would lead to an excessive number of synapses resulting in increased white matter. Interestingly, recent evidence of premature white matter maturation has been found in anterior brain regions of young children with autism (Ben Bashat et al., 2007). Recent work in our laboratory has revealed an increase in the proportional neuropil volume in dorsolateral prefrontal cortex (J. Morgan, personal communication). Relatively modest initial perturbations may have catastrophic effects on later neuronal and 
synaptic organization, driving marked pathological brain overgrowth. Although speculative, these effects may be further compounded over the course of development, as an aberrantly wired brain does not benefit from the same environmental experiences that shape the typically developing brain, further aggravating autistic symptoms and producing additional later brain pathology (for review, see Courchesne et al., 2007).

Given that the onset of autistic symptoms may be associated with early postnatal pathology, early detection and therapy may have compelling effects on outcome. Brain regions that show the greatest degree of enlargement and abnormal growth trajectory in our longitudinal study, including frontal, temporal, and cingulate cortices, have also been reported to display the greatest degree of functional impairment in older children and adults (for review, see Courchesne et al., 2007). These regions are associated with social attention, language development, and emotional reactivity; impairments in these functions are among the first behavioral signs of autism between 1 and 2 years of age (Wetherby et al., 2004; Zwaigenbaum et al., 2005). We have previously reported that brain size in young children with autism is correlated with diagnostic and functional outcome (Akshoomoff et al., 2004). In fact, volumetric brain measurements taken at $\sim 3.4$ years of age correctly classified $96 \%$ of autism spectrum disorder cases and $92 \%$ of typically developing control cases at 5 years of age. In addition, $85 \%$ of autism spectrum cases were correctly classified as low functioning, and $68 \%$ were classified as high functioning based on MRI brain measures alone. If therapeutic intervention can be applied early enough to impact neuroanatomical development, children on the autism spectrum may have substantially better diagnostic and functional outcomes, particularly given the potential for a cascade of downstream pathological alteration.

In summary, we revealed via longitudinal study that the cerebrum in most children with autism is enlarged by 2.5 years of age and that specific subregions have an abnormal growth trajectory. This enlargement and growth pathology is primarily accounted for by the frontal and temporal cortices, and these areas continue to follow an abnormal growth trajectory throughout early childhood. The severity of overgrowth and cerebral regions affected varies by gender; females display a more pronounced abnormal growth profile in more brain regions than males with autism from 2 to 5 years of age. Although it is evident that the brain in the child with autism is undergoing an abnormal growth trajectory throughout early childhood and early brain overgrowth is a consistent and replicated feature of autism, it is important to keep in mind that autism is a heterogeneous disorder and not all children have an enlarged cerebral volume. In fact, no consistent pathology has been found for every child with autism (for review, see Amaral et al., 2008). Imaging studies of brain development on larger populations may begin to address the heterogeneity of the disorder and in the future will likely describe distinct subtypes of autism, each with a specific associated neuroanatomical phenotype. Maximum benefit will be derived from longitudinal studies of even younger populations with additional characterization of underlying genetic abnormalities and other potential biomarkers that are driving overgrowth and the onset of autistic symptoms at this critical time in development.

\section{References}

Akshoomoff N, Lord C, Lincoln A, Courchesne RY, Carper R, Townsend J, Courchesne E (2004) Outcome classification of preschool children with autism spectrum disorders using MRI brain measures. J Am Acad Child Adolesc Psychiatry 43:349-357.
Amaral DG, Schumann CM, Nordahl CW (2008) Neuroanatomy of autism. Trends Neurosci 31:137-145.

American Psychiatric Association (1994) Diagnostic and statistical manual of mental disorders, Ed 4. Washington, DC: American Psychiatric Association.

Ben Bashat D, Kronfeld-Duenias V, Zachor DA, Ekstein PM, Hendler T, Tarrasch R, Even A, Levy Y, Ben Sira L (2007) Accelerated maturation of white matter in young children with autism: a high b value DWI study. Neuroimage 37:40-47.

Bloss CS, Courchesne E (2007) MRI neuroanatomy in young girls with autism: a preliminary study. J Am Acad Child Adolesc Psychiatry 46:515-523.

Carper RA, Moses P, Tigue ZD, Courchesne E (2002) Cerebral lobes in autism: early hyperplasia and abnormal age effects. Neuroimage 16:10381051.

Cohen J, Cohen P, West SG, Aiken, LS (2003) Applied multiple regression/ correlation analysis for the behavioral sciences. Mahwah, NJ: Erlbaum Associates.

Conel JL (1939-1967) The postnatal development of the human cerebral cortex. Cambridge, MA: Harvard UP.

Courchesne E, Karns C, Davis HR, Ziccardi R, Carper R, Tigue Z, Pierce K, Moses P, Chisum HJ, Lord C, Lincoln AJ, Pizzo S, Schreibman L, Haas RH, Akshoomoff N, Courchesne RY (2001) Unusual brain growth patterns in early life in patients with autistic disorder: an MRI study. Neurology 57:245-254.

Courchesne E, Carper R, Akshoomoff N (2003) Evidence of brain overgrowth in the first year of life in autism. JAMA 290:337-344.

Courchesne E, Pierce K, Schumann CM, Redcay E, Buckwalter JA, Kennedy DP, Morgan J (2007) Mapping early brain development in autism. Neuron 56:399-413.

Cox RW (1996) AFNI: software for analysis and visualization of functional magnetic resonance neuroimages. Comp Biomed Res 29:162-173.

Dale AM, Fischl B, Sereno MI (1999) Cortical surface-based analysis. I. Segmentation and surface reconstruction. Neuroimage 9:179-194.

Dawson G, Munson J, Webb SJ, Nalty T, Abbott R, Toth K (2007) Rate of head growth decelerates and symptoms worsen in the second year of life in autism. Biol Psychiatry 61:458-464.

Dementieva YA, Vance DD, Donnelly SL, Elston LA, Wolpert CM, Ravan SA, DeLong GR, Abramson RK, Wright HH, Cuccaro ML (2005) Accelerated head growth in early development of individuals with autism. Pediatr Neurol 32:102-108.

Desikan RS, Segonne F, Fischl B, Quinn BT, Dickerson BC, Blacker D, Buckner RL, Dale AM, Maguire RP, Hyman BT (2006) An automated labeling system for subdividing the human cerebral cortex on MRI scans into gyral based regions of interest. Neuroimage 31:968-980.

Egaas B, Courchesne E, Saitoh O (1995) Reduced size of corpus callosum in autism. Arch Neurol 52:794-801.

Fischl B, Sereno MI, Dale AM (1999) Cortical surface-based analysis. II: Inflation, flattening, and a surface-based coordinate system. Neuroimage 9:195-207.

Fischl B, Liu A, Dale AM (2001) Automated manifold surgery: constructing geometrically accurate and topologically correct models of the human cerebral cortex. IEEE Trans Med Imaging 20:70-80.

Fombonne E (2003) The prevalence of autism. JAMA 289:87-89.

Hazlett HC, Poe M, Gerig G, Smith RG, Provenzale J, Ross A, Gilmore J, Piven J (2005) Magnetic resonance imaging and head circumference study of brain size in autism: birth through age 2 years. Arch Gen Psychiatry 62:1366-1376.

Huttenlocher PR (1979) Synaptic density in human frontal cortex-developmental changes and effects of aging. Brain Res 163:195-205.

Lainhart JE, Piven J, Wzorek M, Landa R, Santangelo SL, Coon H, Folstein SE (1997) Macrocephaly in children and adults with autism. J Am Acad Child Adolesc Psychiatry 36:282-290.

Lord C, Rutter M, Le Couteur A (1994) Autism Diagnostic InterviewRevised: a revised version of a diagnostic interview for caregivers of individuals with possible pervasive developmental disorders. J Autism Dev Disord 24:659-685.

Lord C, Risi S, Lambrecht L, Cook Jr E, Leventhal B, DiLavore P, Pickles A, Rutter M (2000) The Autism Diagnostic Observation Schedule-Generic: a standard measure of social and communication deficits associated with the spectrum of autism. J Autism Dev Disord 30:205-223.

Mraz KD, Green J, Dumont-Mathieu T, Makin S, Fein D (2007) Correlates 
of head circumference growth in infants later diagnosed with autism spectrum disorders. J Child Neurol 22:700-713.

Mullen EM (1995) Mullen Scales of Early Learning, AGS Edition. Circle Pines, MN: American Guidance Service.

Redcay E, Courchesne E (2005) When is the brain enlarged in autism? A meta-analysis of all brain size reports. Biol Psychiatry 58:1-9.

Schumann C, Barnes CC, Lord C, Courchesne E (2009) Amygdala enlargement in toddlers with autism related to severity of social and communication impairments. Biol Psychiatry 66:942-949.

Sparks BF, Friedman SD, Shaw DW, Aylward E, Echelard D, Artru AA, Maravilla KR, Giedd JN, Munson J, Dawson G, Dager SR (2002) Brain structural abnormalities in young children with autism spectrum disorder. Neurology 59:184-192.
Sparrow S, Balla D, Cicchetti D (1984) Vineland scales of adaptive behavior: survey form manual. Circle Pines, MN: American Guidance Service.

Webb SJ, Nalty T, Munson J, Brock C, Abbott R, Dawson G (2007) Rate of head circumference growth as a function of autism diagnosis and history of autistic regression. J Child Neurol 22:1182-1190.

Weschler D (2002) Weschler preschool and primary scale of intelligence, Ed 3. New York: The Psychological Corporation.

Wetherby AM, Woods J, Allen L, Cleary J, Dickinson H, Lord C (2004) Early indicators of autism spectrum disorders in the second year of life. J Autism Dev Disord 34:473-493.

Zwaigenbaum L, Bryson S, Rogers T, Roberts W, Brian J, Szatmari P (2005) Behavioral manifestations of autism in the first year of life. Int J Dev Neurosci 23:143-152. 